\title{
Zr doping on Lithium Niobate crystals: Raman spectroscopy and Chemometrics
}

\author{
Ninel Kokanyan ${ }^{1,2}$, David Chapron, ${ }^{1,2}$ Edvard Kokanyan ${ }^{3,4}$ and Marc D. Fontana ${ }^{1,2}$ \\ ${ }^{l}$ CentraleSupelec, Laboratoire Matériaux Optiques, Photonique et Systèmes, 2 rue E. Belin, 57070 Metz, France \\ ${ }^{2}$ Université de Lorraine, Laboratoire Matériaux Optiques, Photonique et Systèmes, EA-4423, 2 rue E. Belin, 57070 \\ Metz, France \\ ${ }^{3}$ IInstitute for Physical Research, National Academy of Sciences of Armenia, 0203, Ashtarak, Armenia \\ ${ }^{4}$ Armenian State Pedagogical University After Kh. Abovyan, Tigran Mets Ave., 17, Yerevan, Armenia
}

Raman measurements were investigated on $\mathrm{Zr}$ - doped lithium niobate $\mathrm{LiNbO}_{3}$ crystals with different concentrations. Spectra were treated by fitting procedure and principal component analysis which both provide results consistent with each other. The concentration dependence of the frequency of the main low-frequency optical phonons gives an insight of site incorporation of $\mathrm{Zr}$ ions in the host lattice. The threshold concentration of about $2 \%$ is evidenced, confirming the interest of $\mathrm{Zr}$ doping as an alternative to $\mathrm{Mg}$ doping for the reduction of the optical damage in lithium niobate.

\section{Introduction}

A strong limitation to the applications of congruent commercial $\mathrm{LiNbO}_{3}(\mathrm{LN})$ crystals in optical parametric oscillators and electro- optic devices comes from the fact that, under illumination with visible or near-infrared light, there are semi-permanent changes in the index of refraction of the crystal, due to the photorefractive (PR) effect [1]. This so-called "optical damage", causing beam distortion, dramatically diminishes the use of LN in devices. This drawback and limitation exist unless some strategy to reduce the photorefractive effect is implemented. The optical damage resistance (ODR) can be increased considerably by changing the LN crystal composition from congruent to stoichiometric [2-4] and/or by adding into LN lattice an appropriate non-photorefractive dopant [1-5]. Among the ODR dopants that have been tested, the most utilized is, nowadays, $\mathrm{MgO}$ that is known to be efficient in molar concentrations above $5 \mathrm{~mol} \%$ [6]. A problem remains with this dopant owing to difficulties to grow large high optical quality $\mathrm{MgO}$ doped LN crystals. Recently, a set of tetravalent impurity ions $\left(\mathrm{Hf}^{4+}, \mathrm{Sn}^{4+}\right.$, $\mathrm{Zr}^{4+}$ ) as new ODR ions were checked [7-11]. It was shown that for these ions, the concentration threshold for ODR can be strongly reduced. In particular, a sample with $2 \mathrm{~mol} \% \mathrm{ZrO}_{2}$ can withstand a light intensity of $2 \times 10^{7} \mathrm{~W} / \mathrm{cm}^{2}$ of $514 \mathrm{~nm}$ laser. The ODR is therefore 40 times larger than that of $6.5 \% \mathrm{MgO}$ [11]. In addition, it was shown that the non-linear optical-and electro-optical coefficients are preserved $[12,13]$ in $\mathrm{Zr}$-doped LN, so that this crystal is promising for applications of frequency conversion and laser modulation. As a consequence, $\mathrm{Zr}$ - doped LN with a threshold concentration of around 2-3mol\% [14]. can be a good alternative to stoichiometric and/or Mg- doped crystal for reduction of the photorefractive effect. According to Kong et al. [11] the main question remains about the reason of the small threshold achieved with Zr doping, compared with other ODR dopant ions. In other terms the microscopic mechanism of incorporation of $\mathrm{Zr}$ in LN lattice is unknown and the control and improvement of this material requires the understanding of the substitution process. The threshold refers to the concentration above which the OD or PR largely decreases and is generally associated in congruent LN crystal to a complete removal of $\mathrm{Nb}$ antisites (i.e. the $\mathrm{Nb}$ ions going to the Li-site) [1]. When doping, the impurity ion can go to the site $\mathrm{A}$ 
of native $\mathrm{Li}$ ions, or the site $\mathrm{B}$ of native $\mathrm{Nb}$ ions, so that in doped $\mathrm{LN}$ materials the $\mathrm{PR}$ effect can increase or decrease, according to the site occupation of the dopant.

In previous studies we have shown that Raman spectroscopy (RS) can be a useful probe of dopant ion [15, 16]. A shift of line position (optical phonon frequency) and/or linewidth (phonon damping) of some Raman lines can be thus related to incorporation of defects in the host lattice.

In the present work the $\mathrm{Zr}$-doped LN crystals with different concentrations are investigated by means of Raman micro-probe, in order to have an insight of the sites occupied by $\mathrm{Zr}$ ions in the structure of LN and thus an understanding of the threshold concentration required for the reduction of the photorefractive effect as well.

Among all Raman lines we paid attention to those which can be used as the discriminating markers of sites A or B [17]. Thus, the lowest-frequency $\mathrm{E}\left[\mathrm{TO}_{1}\right]$ and $\mathrm{A}_{1}\left[\mathrm{TO}_{1}\right]$ Raman lines, which are associated with the motion of $\mathrm{Nb}$ against oxygen octahedron, can be suitably probe the site $\mathrm{B}$ occupied by native $\mathrm{Nb}$ ions. The line $\mathrm{A}_{1}\left[\mathrm{TO}_{2}\right]$ corresponds to the out- of- phase vibration of $\mathrm{Li}$ and $\mathrm{O}$ and can be therefore used to study the environment of the site $\mathrm{A}$ and changes due to Li ions, $\mathrm{Li}$ vacancies and $\mathrm{Nb}$ antisites $[17,18]$.

The concentration of $\mathrm{Zr}$ samples in samples under study is small (below $2.5 \%$ ) i.e. much lower than the content of dopant ions used in previous works $[15,16]$. Furthermore, the difference of concentrations between two "consecutive" samples in the $\mathrm{Zr}$ series under study is very small, so that only a subsequent very small change in Raman spectrum is expected. Chemometrics are technique able to evidence very small changes in spectra [19] and are therefore used here to support the exploitation of Raman spectra, and the dependences of vibrational modes on $\mathrm{Zr}$ content. Finally, the behavior of main modes vs $\mathrm{Zr}$ is used to derive the incorporation mechanism of $\mathrm{Zr}$ in the $\mathrm{LN}$ lattice

\section{Experimental results and analysis}

The crystals were grown by Czochralski method from congruent melts to which appropriate amounts of zirconium oxide $\left(\mathrm{ZrO}_{2}\right)$ were added. A set of samples with concentrations of $\mathrm{ZrO}_{2}$ equal to $0.625,1.00,1.25,1.50,2.00$ and $2.50 \mathrm{~mol} \%$, were prepared and denoted respectively as LNZr0.625, LNZr1, ... Given concentrations correspond to the content of $\mathrm{ZrO} 2$ in the melt and are very close to those in the crystals. The relative amount of $\mathrm{Zr}$ in the crystals, and the presence of impurities other as $\mathrm{Zr}$ were checked with X-ray fluorescence. Raman measurements were carried out by means of a Horiba-Aramis spectrometer with an absolute spectral resolution of $1 \mathrm{~cm}^{-1}$ and diffraction grating of $1200 \mathrm{~g} . \mathrm{mm}^{-1}$. The $633 \mathrm{~nm}$ of a He-Ne laser with intensity of $1.27 * 10^{7} \mathrm{~W} / \mathrm{m}^{2}$ was used as the exciting line and the scattered radiation was detected by a CCD camera (200 pixels).

$\mathrm{E}\left[\mathrm{TO}_{1}\right]$ and $\mathrm{A}_{1}\left[\mathrm{TO}_{1}+\mathrm{TO}_{2}\right]$ spectra were recorded in the $\mathrm{Y}(\mathrm{XZ}) \overline{\mathrm{Y}}$ and $\mathrm{Y}(\mathrm{ZZ}) \overline{\mathrm{Y}}$ backscattering configurations respectively $[17$, 20]. Raman spectra were carried out at room temperature and low temperature $\left(-180^{\circ} \mathrm{C}\right)$ as well in order to obtain more resolved lines. Indeed, lines at room or higher temperature are broader and asymmetric rendering more difficult any spectra exploitation [21]. 


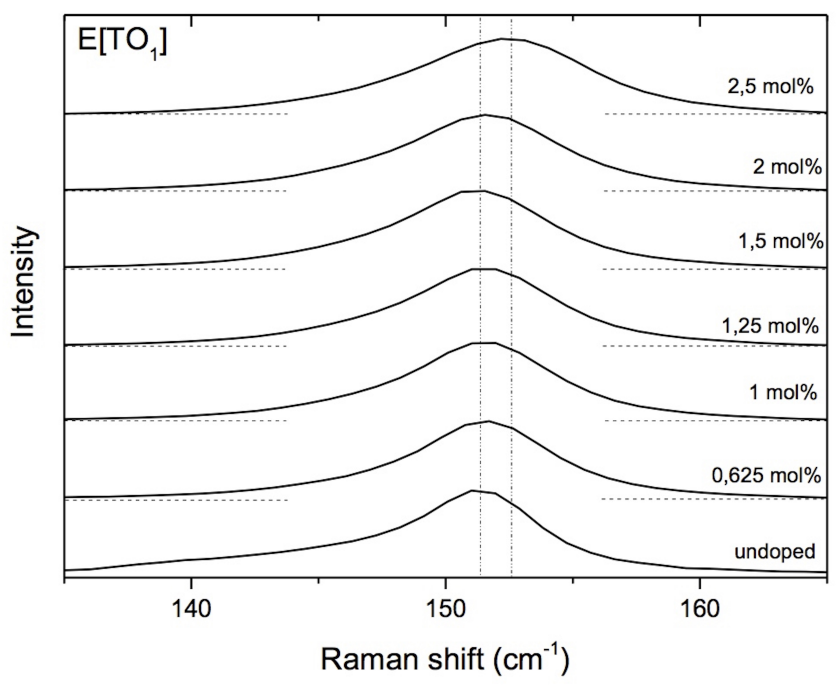

FIG 1. Raman spectra at $-180^{\circ} \mathrm{C}$ of lowest frequency $\mathrm{E}\left[\mathrm{TO}_{1}\right]$ mode for different concentrations of $\mathrm{Zr}$ in $\mathrm{Zr}$ - doped Lithium Niobate crystals. Vertical lines correspond to the frequency of undoped LN and LNZr2.5 samples.

$\mathrm{E}\left[\mathrm{TO}_{1}\right]$ Raman spectra on undoped and various $\mathrm{Zr}$ doped $\mathrm{LN}$ crystals are reported in figure 1. A continuous broadening is noted with increasing $\mathrm{Zr}$ content while a small shift of peak maximum is observable for LNZr2 and LNZr2.5 samples as compared with all others. Spectra were fitted to damped harmonic oscillators in order to derive the frequency and the damping of phonons $\mathrm{E}\left[\mathrm{TO}_{1}\right], \mathrm{A}_{1}\left[\mathrm{TO}_{1}\right]$ and $\mathrm{A}_{1}\left[\mathrm{TO}_{2}\right]$. Results are reported in figure 2 .

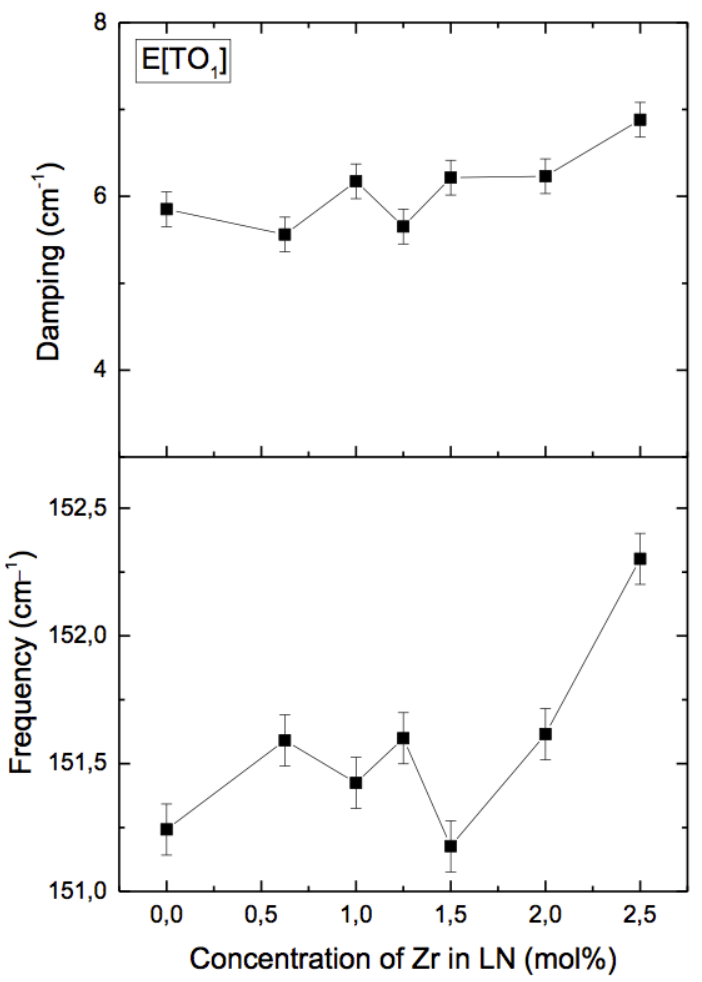

(a)

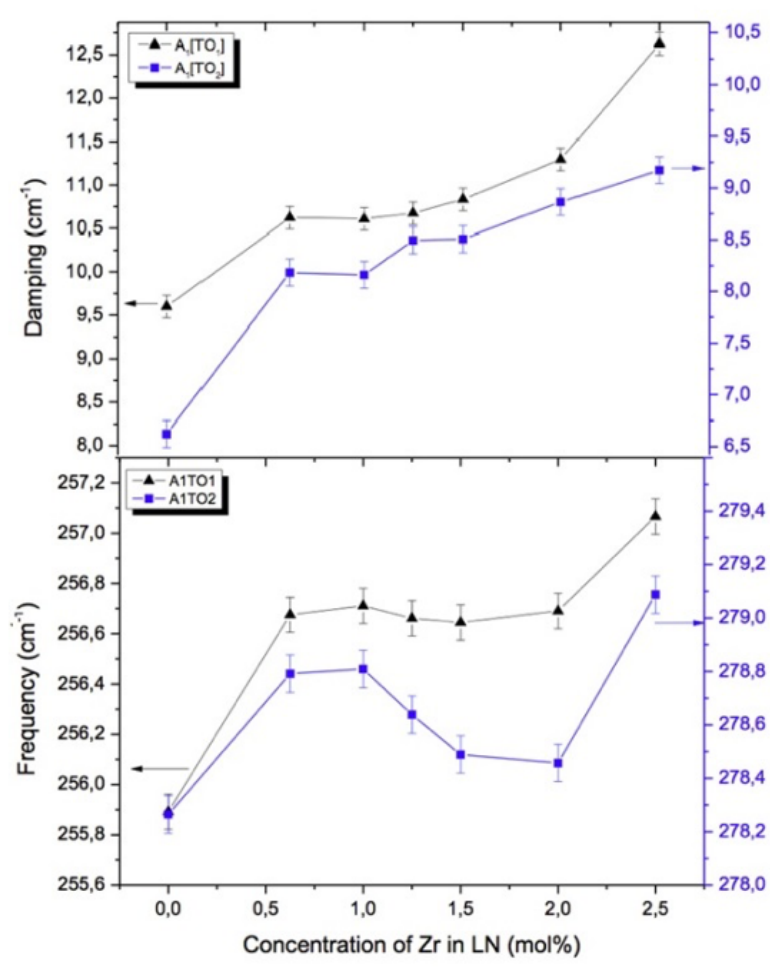

(b)

FIG 2. Concentration dependence of the frequency and damping of $E\left[\mathrm{TO}_{1}\right]$ (a) $\mathrm{A}_{1}\left[\mathrm{TO}_{1}\right]$ and $\mathrm{A}_{1}\left[\mathrm{TO}_{2}\right]$ (b) phonons. 
One can notice that the damping for each phonon under study continuously increases with doping, reflecting a growing disorder in the LN lattice, whereas the frequency exhibits a minimum value for 1.5 or $2 \% \mathrm{Zr}$, and then increases for larger concentrations. This anomaly in the behavior of dependence on $\mathrm{Zr}$ content needed to be confirmed since only small changes of frequency were observed. Therefore, Raman spectra were additionally treated by principal component analysis (PCA), in order to evidence their relative variation with $\mathrm{Zr}$ concentration. Indeed, PCA is a statistical method commonly used for data classification and can be applied to the spectra analysis allowing to express their relative variability by projection on orthogonal components [22$25]$.

Performing a PCA on spectra we obtain the projection values of sample on each PC. The score corresponds to the relative weight of each spectrum to one component and thus gives an idea of the relative change between different samples. Loadings represent the new base, which are representative to the variation on each spectrum

$$
\text { Spectrum }=\langle S\rangle+s_{1} P C_{1}+s_{2} P C_{2}+\cdots+\varepsilon
$$

Where $<\mathrm{S}>$ is the average spectrum, $\mathrm{s}_{\mathrm{i}}$ are scores, $\mathrm{PC}_{\mathrm{i}}$ are loadings and $\varepsilon$ are residual values. In our investigations $\mathrm{PCA}$ was performed using the Unscrambler10.3 software [26]. The analysis was performed after performing a standard normal variant pretreatment on the spectra utilizing the same software. The transformation is applied to each spectrum individually by subtracting the mean spectrum and scaling with the spectrum standard deviation. This means that it normalizes the intensity and corrects the baseline of all spectra.

In figure 3 are plotted different results derived from PCA applied to the spectra reported in figure 1. The components PC1 and PC2 provide 96 and 3\% of the entire signal, so that they nearly reflect the whole variance. Whereas PC1 gives the change in intensity, PC2 reveals the change in the maximum peak position, since it behaves as the first derivative. The component related to line position shift (here PC2) is only used hereafter to exploit and interpret Raman data. 


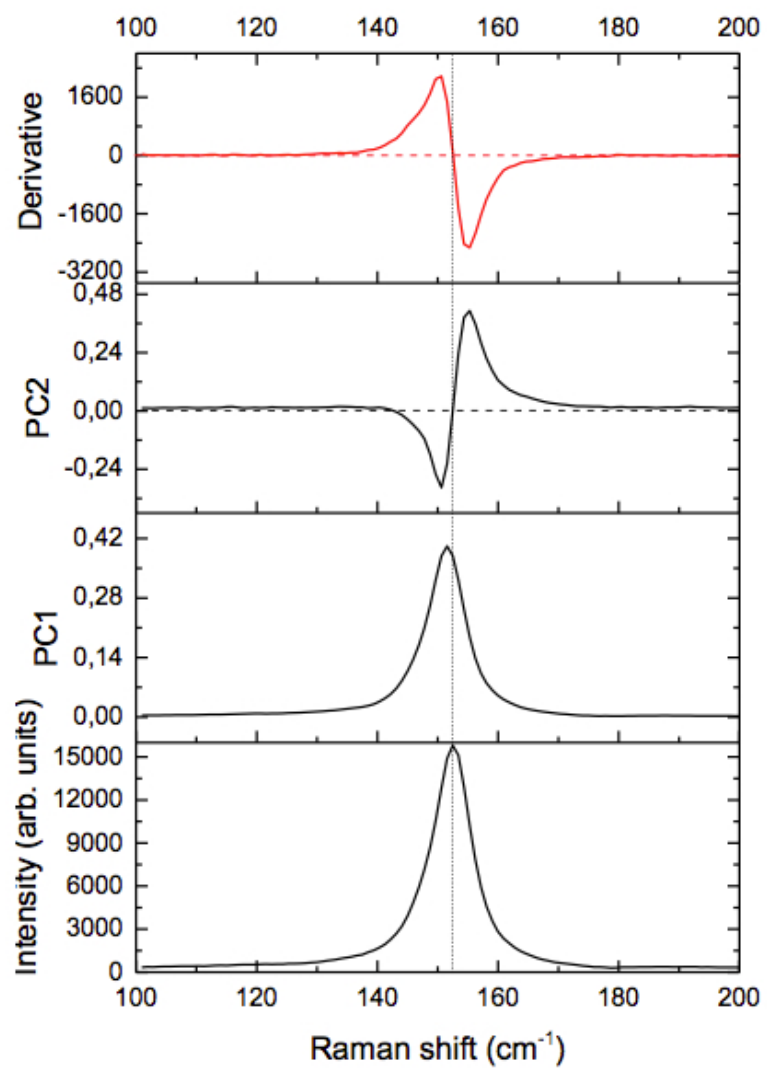

FIG 3. Loadings of $\mathrm{PC} 1$ and $\mathrm{PC} 2$ of PCA performed on the spectra (range of $\left.100 \mathrm{~cm}^{-1}-200 \mathrm{~cm}^{-1}\right)$ obtained at low temperature $\left(-180^{\circ} \mathrm{C}\right)$, for the $\mathrm{Y}(\mathrm{ZX}) \overline{\mathrm{Y}}$ configuration. The spectrum of $2 \mathrm{~mol} \% \mathrm{Zr}$ (at the bottom) and its derivative (at the top) are shown for comparison.

Scores obtained for PC2 for all spectra (or $\mathrm{Zr}$ contents) are plotted in fig 4 and compared with the dependence of E[TO $\left.{ }_{1}\right]$ frequency as derived from usual fit procedure of spectra. A similar comparison between score deduced from PC analysis and phonon frequency, for $\mathrm{A}_{1}\left[\mathrm{TO}_{1}\right]$ mode is reported in figure 5 . In both cases the behaviors with $\mathrm{Zr}$ content are remarkably similar. This consistency between results derived from two completely different analysis processes from the same Raman spectra, corroborates the dependences of phonon frequency given above and renders more reliable the interpretation of these changes with $\mathrm{Zr}$ content.

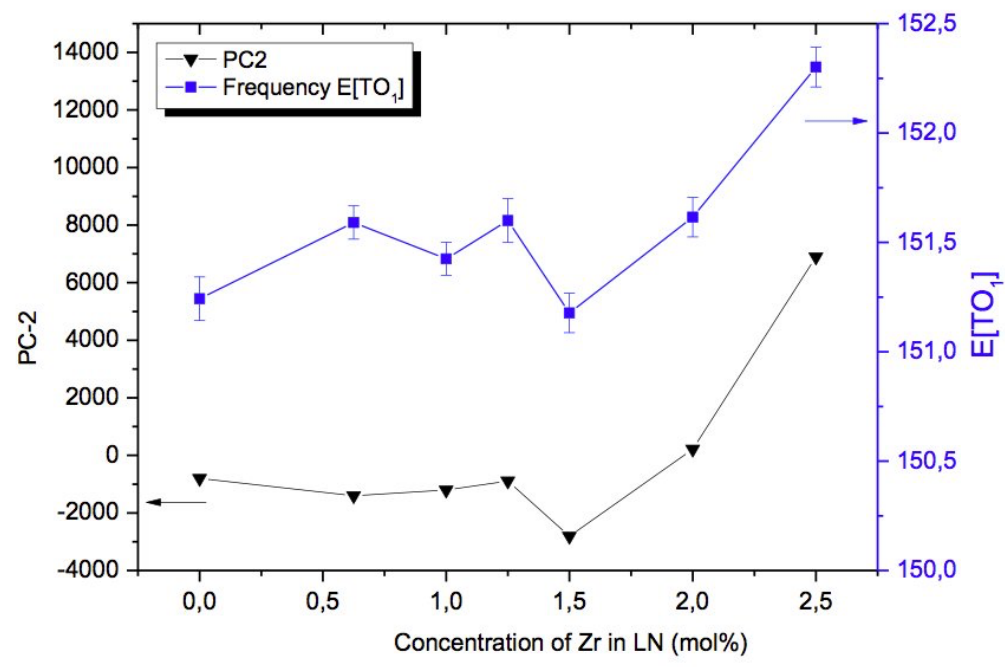

FIG 4. Frequency of $\mathrm{E}\left[\mathrm{TO}_{1}\right]$ mode and score of $\mathrm{PC} 2$ as a function of the $\mathrm{Zr}$ concentration in Lithium Niobate. 


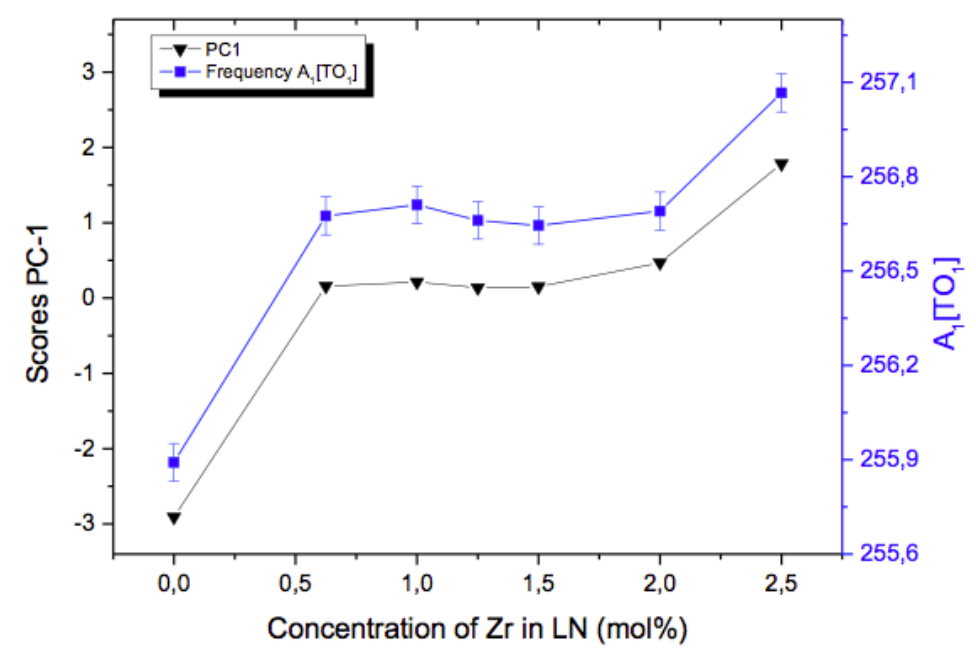

FIG 5. Frequency of $\mathrm{A}_{1}\left[\mathrm{TO}_{1}\right]$ mode and score of $\mathrm{PC} 1$ as a function of the $\mathrm{Zr}$ concentration in Lithium Niobate.

\section{Interpretation and Conclusion}

We are now able derive the mechanism of incorporation of $\mathrm{Zr}$ ions in the LN lattice from the dependences of the frequency of $\mathrm{E}\left[\mathrm{TO}_{1}\right], \mathrm{A}_{1}\left[\mathrm{TO}_{1}\right]$ and $\mathrm{A}_{1}\left[\mathrm{TO}_{2}\right]$ plotted in figure 2. We remind that the modes $\mathrm{E}$ and $\mathrm{A}_{1}\left[\mathrm{TO}_{1}\right]$ mainly involve $\mathrm{B}$ site, while the phonon $\mathrm{A}_{1}\left[\mathrm{TO}_{2}\right]$ probes the site $\mathrm{A}$. Furthermore, $\mathrm{A}_{1}$ phonons correspond to ionic motions along the ferroelectric $\mathrm{c}$ axis, while E phonons are polarized in the plane normal to c. When they are incorporated in the $\mathrm{LN}$ lattice, $\mathrm{Zr}$ ions can at first replace $\mathrm{Nb}$ antisites on sites $\mathrm{A}$, pushing $\mathrm{Nb}$ on going to their intrinsic $\mathrm{B}$ sites. As a consequence, the $\mathrm{A}_{1}\left[\mathrm{TO}_{2}\right]$ mode frequency increases due to a strengthening of $\mathrm{A}-\mathrm{O}$ bond since ionic radius of $\mathrm{Zr}^{4+}$ is larger than $\mathrm{Nb}^{5+}$, whereas the frequencies of $\mathrm{A}_{1}\left[\mathrm{TO}_{1}\right]$ and $\mathrm{E}\left[\mathrm{TO}_{1}\right]$ are rising as well, involving stronger $\mathrm{B}-\mathrm{O}$ bond.

A more increase of the $\mathrm{Zr}$ concentration leads to an enhancement of the occupation of $\mathrm{Li}$ sites. When $\mathrm{Zr}$ replaces $\mathrm{Li}$, as the mass of $\mathrm{Zr}$ ion is larger than this of $\mathrm{Li}$ ion, the frequency of $\mathrm{A}_{1}\left[\mathrm{TO}_{2}\right]$ mode decreases for concentration varying between 1 and $2 \mathrm{~mol} \%$. In this range the frequency of phonons $\mathrm{A}_{1}\left[\mathrm{TO}_{1}\right]$ and $\mathrm{E}\left[\mathrm{TO}_{1}\right]$ is nearly constant. Finally, for $2.5 \mathrm{~mol} \%$ concentration the frequencies of $\mathrm{A}_{1}\left[\mathrm{TO}_{1}\right], \mathrm{E}\left[\mathrm{TO}_{1}\right]$ and $\mathrm{A}_{1}\left[\mathrm{TO}_{2}\right]$ modes increase again. This can be attributed to the occupation of the $\mathrm{Zr}$ ions on both sites A and B as well, rendered more disordered the LN structure as reflected by the phonon damping.

It can be mentioned that $\mathrm{Zr}$ introduction is accompanied by a large increase of $\mathrm{A} 1$ phonon damping. This means that the $\mathrm{Zr}$ ion is slightly displaced along $\mathrm{c}$ axis with respect of native $\mathrm{Li}$ and $\mathrm{Nb}$ sites.

Our results show that $2 \%$ is a critical content above which the mechanism of $\mathrm{Zr}$ ion incorporation in LN lattice strongly changes. As shown by the behavior of phonons $\mathrm{A}_{1}\left[\mathrm{TO}_{1}\right]$ and $\mathrm{E}\left[\mathrm{TO}_{1}\right]$ which are both related to $\mathrm{Nb}$ site, the concentration $2 \%$ corresponds to the total remove of $\mathrm{Nb}$ antisites and therefore to the threshold of optical damage resistance. These studies confirm the interest of $\mathrm{Zr}$ doping for reducing the optical damage of LN.

It was shown that PCA can be useful to highlight small variations between spectra. More generally it is a fast method comparing to classical fitting method, and can be specially used in the case of a big number of samples and/or spectra in order to make a sort between samples before starting fitting procedure. 


\section{References}

[1] T.R.Volk and M.Woehlecke, Lithium Niobate. Defects, Photorefraction and Ferroelectric Switching, Springer Series in Materials Science (2008).

[2] G.I. Malovichko, V.G. Grachev, E.P. Kokanyan, O.F. Schirmer, K. Betzler, B. Gather, F. Jermann, S. Klauer, U. Schlarb, M. Wöhlecke, Appl. Phys. A 56, 103 (1993).

[3] K.Kitamura, J.K.Yamamoto, N.Iyi, S.Kirnura, J. of Crystal Growth 116, 327 (1992).

[4] M.Fontana, K.Chah, M.Aillerie, R.Mouras, P.Bourson, Optical Materials 16, 111 (2001).

[5] A.M.Petrosyan, R.K.Hovsepyan, E.P.Kokanyan, R.S.Feigelson, Proc. SPIE 4060, 106 (2000).

[6] G. Zhong, J.Jin, Z.Wu, J. Opt. Soc. Am 70, 631 (1980).

[7] E.P.Kokanyan, L.Razzari, I.Cristiani, V.Degiorgio and J.B.Gruber, Appl. Phys. Letters 84, 1880 (2004).

[8] S.Li, S.Liu, Y.Kong, D.Deng, G.Gao, Y.Li, H.Gao, L.Zhang, Z.Hang, S.Chen J. of Physics: Condensed Matter 18, 13 (2006).

[9] P.Minzioni, I.Cristiani, J.Yu, J.Parravicini, E.P.Kokanyan, and V.Degiorgio, Optics Express 15, 14171 (2007).

[10] L.Wang, S.Liu, Y.Kong, S.Chen, Z.Huang, L.Wu, R.Rupp, J.Xu, Optics Letters 35, 883 (2010).

[11] Y. Kong, Sh. Liu, Y. Zhao, H. Liu, Sh. Chen, J. Xu, Appl. Phys. Letters 91, 081908 (2007).

[12] G. Nava, P. Minzioni, W. Yan, J. Parravicini, D. Grando, E. Musso, I. Cristiani, N. Argiolas, M. Bazzan, M. V. Ciampolillo, A. Zaltron, C. Sada, V. Degiorgio, Optical Materials Express 1, 270 (2011).

[13] M. Abarkan, M. Aillerie, N. Kokanyan, C. Teyssandier, E. Kokanyan, Optical Materials Express 4, 179 (2014).

[14] N. Argiolas, M. Bazzan, M. V. Ciampolillo, P. Pozzobon, C. Sada, L. Saoner, A. M. Zaltron, L. Bacci, P. Minzioni, G. Nava, J. Parravicini, W. Yan, I. Cristiani, V. Degiorgio, J. of Appl. Phys. 108, 093508 (2010).

[15] R Mouras, M D Fontana, P Bourson, A V Postnikov, J. Phys. Condens. Matter 12, 5053 (2000).

[16] R. Hammoum, M.D. Fontana, M. Gilliot, P. Bourson, E.P. Kokanyan, Solid State Communications 149, 1967 (2009).

[17] M.D.Fontana, P.Bourson, Appl. Phys. Rev. 2, 040602 (2015).

[18] V. Caciuc, A.V. Postnikov, G. Borstel, Phys. Rev. B 61, 8806 (2000).

[19] H. Mark, J. Workman, Chemometrics in Spectroscopy, Elsevier, London, UK (2007).

[20] A.Ridah, P.Bourson, M.D.Fontana, G.Malovichko J. of Phys.: Condensed Matter 9, 44 (1997).

[21] N. Kokanyan, D. Chapron, M. D. Fontana, Appl. Phys. A 117, 1147 (2014).

[22] JE. Jackson, Hoboken, A User's Guide to Principal Components Analysis, NJ:John Wiley\&Sons (2003).

[23] I.T.Jolliffe, Principal Component Analysis, Springer Series in Statistics (2002).

[24] T.R.Brown, R. Stoyanova, J. of Magnetic Resonance B 112, 31 (1996).

[25] H. Witjes, M. van den Brink, W.J. Melssen, L.M.C. Buydens, Chemometrics and Intelligent Laboratory Systems 52, 105 (2000).

[26] http://www.camo.com/rt/Products/Unscrambler/unscrambler.html 